\title{
Neural Pathway
}

National Cancer Institute

\section{Source}

National Cancer Institute. Neural Pathway. NCI Thesaurus. Code C13079.

The route in which a nerve impulse travels through a series of linked axons to connect different regions within the central nervous system or to convey information from the peripheral nervous system to the brain. 